\title{
Discrete Lagrange equations for reacting thermofluid dynamics in arbitrary Lagrangian-Eulerian frames
}

\author{
Charles R. Hean, Eric P. Fahrenthold* \\ Department of Mechanical Engineering, University of Texas, Austin, TX 78712, USA
}

\begin{abstract}
Interest in the development of multiscale methods has focused attention on the marked differences in the numerical modeling techniques typically applied at different scales. Most continuum dynamics models construct approximate solutions to partial differential equations, while most nanoscale models employ a discrete Hamiltonian approach. Previous research has demonstrated that the introduction of entropies or internal energies as generalized coordinates, along with separation of the discretization and model formulation steps, allows general thermomechanical models to be developed, at the continuum scale, using a nonholonomic Hamiltonian or Lagrange equation formulation. With the introduction of additional state variables and nonholonomic constraints, the latter work may be further extended in order to model reacting systems. Employing a finite element interpolation and a Lagrangian, an Eulerian, or an ALE mesh, the new formulation has been validated by solving several one-dimensional reacting shock physics problems. The result is a continuum dynamics modeling approach highly compatible with the discrete energy methods normally used at the nanoscale.
\end{abstract}

Keywords: Lagrange's equations, ALE, reacting systems, detonation

\section{Introduction}

In previous work the authors and co-workers $[1,2,3]$ have developed new discrete energy methods for continuum fluid dynamics modeling. Employing entropies or internal energies as generalized coordinates, and a finite element interpolation of the flow field, the cited works formulated discrete Hamiltonian or Lagrange equation formulations for viscous compressible fluid dynamics in Lagrangian, Eulerian, and arbitrary Lagrangian-Eulerian (ALE) frames. The ODE models were developed without reference to any partial differential equations, and were validated by solving a set of shock physics problems. The latter work established the feasibility of applying typical nanoscale modeling methods to thermomechanical continuum dynamics simulations, for both open and closed systems, and therefore offers opportunities for the development of multiscale simulation techniques based on a unified energy-based modeling methodology. This paper extends the aforementioned work to reacting flows, including the strong chemical-thermomechanical coupling associated with detonations.

The chemical dynamics included in a reacting flow model is normally quantified by modeling processes which occur on a scale much smaller than that required to model the fluid dynamics. At the molecular level energy based modeling methods are the norm [4], while at the system level the use of energy methods is relatively rare [5]. Both finite difference $[6,7]$ and finite element methods

\footnotetext{
${ }^{*}$ Corresponding author Tel.:+1-512-471-3064; fax: +1-512-471-7682.

Email address: epfahren@mail.utexas.edu (Eric P. Fahrenthold)
} 
have been employed to model reacting systems. Explicit methods $[8,9,10]$ are typically used to model fast flows, such as detonations, while implicit methods [11] have been effectively employed in modeling slower systems. Oran and Boris [12] and Powers [13] review various methods used in numerical simulation of reactive flow and detonations, including multi-scale modeling.

In general the introduction of finite-rate chemistry leads to significant complications. Additional state variables are needed, one for each chemical species, as well as species continuity relations with source terms. The choice of chemical kinetic model, including the number of modeled reactions and their rate constants, largely determines computational cost. For example in combustion chemistry, computational requirements increase with the size of the fuel molecule. Hydrogen-air combustion models typically involve approximately 10 species and 30-60 reaction equations, if nitrogen is considered inert. Including nitrogen chemistry can double the number of species and reactions. Methane and ethane based models may again double the number of species and reactions, while octane models may include 800 species and 3000 reactions [12]. At very high temperatures, modeling the free electrons in an ionizing flow may be required. Stiffness problems may arise in systems involving fast reactions of species with small concentrations $[12,14,6]$. In view of these complications, simulations of detonation are often limited to one spatial dimension [13, 15, 16, 17], sometimes as a prelude to more expensive two and three dimensional simulations [18]. In some previous work, the assumption of simplified one-step kinetics (two species and a single, irreversible reaction equation) has allowed for very efficient modeling of reacting shocks [12, 19, 20, 21, 22, 23]. It has also allowed for the study of unstable 1-D detonations [22, 24, 18, 25]. In these models resolution requirements may influence predictions of detonation stability $[15,17]$; the same may be true when more detailed kinetics $[26,27]$ are included. Modeled instabilities are often described as pulsating or galloping, characterized by regular, short-period, low-amplitude oscillations.

The formulation of the system level model developed in this paper may be outlined as follows. First a simple finite-element interpolation is introduced and the state variables are chosen (they include the mesh coordinates). Having adopted an ALE frame of reference, a set of constraints describing the mesh motion is then specified [3]. Then the kinetic and potential energies are formulated, the latter as a simple sum of the element internal energies. Next, the conservation of mass and energy relations and the species evolution equations are introduced, as nonholonomic constraints. A virtual work expression is then developed, which includes the effects of momentum and energy convection. Finally the canonical Lagrange equations are introduced, and are specialized to obtain the final state equations for the system. To validate the model, a number of example problems are solved, which include: (1) Eulerian, Lagrangian, and ALE meshes, (2) both open and closed systems, (3) simplified as well as relatively detailed chemical kinetics, and (4) stable as well as unstable pulsating overdriven detonations. Note that species diffusion effects are neglected, as is typically the case for shocked flow and detonation simulations $[28,12,7]$.

\section{Discretization}

The finite element interpolation adopted here employs piecewise constant velocity gradients, with mass densities and internal energy densities also uniform on an element. In the one dimensional models described in this paper, these elements are two-noded lines; they are of course three-noded triangles or four-noded tetrahedra in two and three dimensions. On external boundaries open to flow, the total pressure and total enthalpy are taken to be piecewise constant.

The system level model is composed of $n_{e}$ elements and $n_{n}$ nodes. The extensive state variables for each element are the total mass $m^{(i)}$, the total internal energy $U^{(i)}$, and the species masses $s_{k}^{(i)}$ $\left(k=1 \ldots N_{s}-1\right)$ for a mixture of $N_{s}$ chemical species where

$$
\sum_{k=1}^{N_{s}} s_{k}^{(i)}=m^{(i)}
$$


In one dimension the global mass, species mass, and internal energy vectors are (with superscript $T$ the transpose)

$$
\mathbf{m}=\left[\begin{array}{llll}
m_{1} & m_{2} & \ldots & m_{n_{e}}
\end{array}\right]^{T} \quad, \quad \mathbf{s}=\left[\begin{array}{lll}
\mathbf{s}^{(1) T} \mathbf{s}^{(2) T} \ldots \mathbf{s}^{\left(n_{e}\right) T}
\end{array}\right]^{T} \quad, \quad \mathbf{U}=\left[\begin{array}{llll}
U_{1} & U_{2} & \ldots & U_{n_{e}}
\end{array}\right]^{T}
$$

while the global vectors for the velocity $\mathbf{v}$ and mesh coordinates $\mathbf{w}$ are

$$
\mathbf{v}=\left[\begin{array}{llllll}
v_{1} & v_{2} & \ldots & v_{n_{n}}
\end{array}\right]^{T} \quad \text { and } \quad \mathbf{w}=\left[\begin{array}{lllll}
w_{1} & w_{2} & \ldots & w_{n_{n}}
\end{array}\right]^{T}
$$

The equation of state is assumed to take the form

$$
\begin{aligned}
e^{(i)} & =e^{(i)}\left(\rho^{(i)}, \theta^{(i)}, Y_{1}^{(i)} \ldots Y_{N_{s}-1}^{(i)}\right) \\
P^{(i)} & =P^{(i)}\left(\rho^{(i)}, \theta^{(i)}, Y_{1}^{(i)} \ldots Y_{N_{s}-1}^{(i)}\right)
\end{aligned}
$$

where $e^{(i)}$ is the mixture internal energy per unit mass, $P^{(i)}$ is the mixture thermodynamic pressure, $\rho^{(i)}$ is the mixture mass density, $\theta^{(i)}$ is the mixture temperature, and the $N_{s}$ species mass fractions for each element $\left(Y_{k}^{(i)}\right)$ are

$$
Y_{k}^{(i)}=\frac{s_{k}^{(i)}}{m^{(i)}} \quad\left(k=1 \ldots N_{s}-1\right) \quad, \quad Y_{N_{s}}^{(i)}=1-\sum_{k=1}^{N_{s}-1} Y_{k}^{(i)}
$$

All species are assumed to be at the same temperature, and the mixture component properties are assumed to be at most temperature-dependent. The equation of state may be analytical or tabulated [14].

Since the model assumes an ALE mesh, the nodal velocities are implicit functions of the nodal coordinates. In addition, since the velocity gradients are uniform on each element, the velocity gradient field is discontinuous across element boundaries. At each node, the velocity gradient is defined to be to the average of the velocity gradients on the neighboring elements. A system level matrix of velocity gradients $(\mathbf{L})$, defined by

$$
\mathbf{L}=\frac{\partial \mathbf{v}}{\partial \mathbf{w}}
$$

will be used to describe certain terms in the the virtual work.

\section{Adaptive mesh}

In the ALE model developed here the mesh coordinates are described by evolution equations (no mesh remap $[12,26,27]$ is introduced). These evolution equations are the nonholonomic constraints

$$
\dot{\mathbf{w}}=\mathbf{X v}
$$

where an Eulerian mesh is defined by $\mathbf{X}=\mathbf{0}$ and a Lagrangian mesh is defined by $\mathbf{X}=\mathbf{I}$. In general, the coefficient matrix $\mathbf{X}$ may be specified as a function of time or the state variables.

\section{Kinetic and potential energy}

Since the system level model will be based on a Lagrange equation $[29,30]$ formulation, expressions for the system kinetic co-energy [31] and system potential energy [32] must be defined. The kinetic co-energy is the sum of the element kinetic co-energies

$$
\mathcal{T}^{*}=\sum_{i=1}^{n_{e}} \mathcal{T}^{*(i)}=\frac{1}{2} \sum_{i=1}^{n_{e}} m^{(i)} \mathbf{v}^{(i) T} \hat{\mathbf{M}}^{(i)} \mathbf{v}^{(i)}=\frac{1}{2} \mathbf{v}^{T} \mathbf{M} \mathbf{v}
$$


where $\mathbf{v}^{(i)}$ is an element-level vector of nodal velocities, $\hat{\mathbf{M}}^{(i)}$ is a dimensionless element mass matrix, and $\mathbf{M}$ is the global mass matrix. In a Lagrange equation context, the nodal velocities are termed 'quasivelocities' since their integration with respect to time does not define physically meaningful generalized coordinates. Alternative quasivelocities may be selected, for convenience. Here we choose as alternative quasivelocities the nodal momenta (p), so that the kinetic co-energy may be written as

$$
\mathcal{T}^{*}=\frac{1}{2} \mathbf{p}^{T} \mathbf{M}^{-1} \mathbf{p}, \quad \mathbf{p}=\mathbf{M} \mathbf{v}
$$

In open systems the element masses vary with time, so the kinetic co-energy also defines a generalized force

$$
-\mathbf{k}=\frac{\partial \mathcal{T}^{*}}{\partial \mathbf{m}}
$$

which will appear in the canonical Lagrange equations.

The system potential energy is simply the sum of the element internal energies

$$
\mathcal{V}=\sum_{i=1}^{n_{e}} U^{(i)}
$$

As a result, the generalized conservative forces for the system are all either zero or unity

$$
\frac{\partial \mathcal{V}}{\partial \mathbf{U}}=\mathbf{1}, \quad \frac{\partial \mathcal{V}}{\partial \mathbf{m}}=\mathbf{0}, \quad \frac{\partial \mathcal{V}}{\partial \mathbf{s}}=\mathbf{0}, \quad \frac{\partial \mathcal{V}}{\partial \mathbf{w}}=\mathbf{0}
$$

\section{Conservation of mass}

The conservation of mass equations are formulated in an arbitrary Lagrangian-Eulerian frame, hence they depend on the mesh coordinates $\mathbf{w}$ and their time derivatives. The net mass inflow to each element is determined by the relative motion of the material with respect to the mesh

$$
\dot{m}^{(i)}=-\int_{S^{(i)}} \hat{\rho}(\hat{\mathbf{v}}-\dot{\mathbf{w}}) \cdot \hat{\mathbf{n}} d S
$$

for density $\hat{\rho}$ and material velocity $\hat{\mathbf{v}}$ distributions across an element surface $S^{(i)}$ with unit normal vector $\hat{\mathbf{n}}$. In discrete form

$$
\dot{m}^{(i)}=\sum_{j=1}^{n_{s}} \rho^{(i, j)} \mathbf{a}^{(i, j) T}(\mathbf{v}-\dot{\mathbf{w}})
$$

where the mass flux is summed over $n_{s}$ element surfaces, for an upwind mass density at the $j^{\text {th }}$ surface $\rho^{(i, j)}$ and a coefficient vector $\mathbf{a}^{(i, j)}$ which depends in general on the mesh coordinates. The system-level conservation of mass relation is

$$
\dot{\mathbf{m}}=\mathbf{A}(\mathbf{v}-\dot{\mathbf{w}})=\mathbf{A}(\mathbf{I}-\mathbf{X}) \mathbf{M}^{-1} \mathbf{p}
$$

\section{Species mass conservation equations}

The species mass conservation equations include both convection and source terms. With $Y_{k}^{(i, j)}$ denoting an upwind species mass fraction, convection of the $k^{\text {th }}$ species into the $i^{\text {th }}$ element is expressed as

$$
\dot{s}_{k}^{c v c(i)}=\sum_{j=1}^{n_{s}} Y_{k}^{(i, j)} \rho^{(i, j)} \mathbf{a}^{(i, j) T}(\mathbf{v}-\dot{\mathbf{w}}) \quad k=1 \ldots N_{s}-1
$$


At the global level, the species evolution equations are

$$
\dot{\mathbf{s}}=\mathbf{Z}(\mathbf{I}-\mathbf{X}) \mathbf{M}^{-1} \mathbf{p}+\boldsymbol{\omega}
$$

where $\mathbf{Z}$ is a convection coefficient matrix which depends on the species mass fractions and the mixture density, and $\boldsymbol{\omega}$ is a source term. Note that the source terms are locally mass-conservative, so that for each element

$$
\sum_{k=1}^{N_{s}} \omega_{k}^{(i)}=0
$$

As illustrated in the example section, the source terms are frequently state variable dependent.

\section{Internal energy evolution equations}

The internal energy evolution equations include convection, flow work, viscous dissipation, and heat conduction terms. The convection model assumed here again depends on upwind properties (for example specific internal energy $e^{(i, j)}$ )

$$
\dot{U}^{c v c(i)}=\sum_{j=1}^{n_{s}} e^{(i, j)} \rho^{(i, j)} \mathbf{a}^{(i, j) T}(\mathbf{v}-\dot{\mathbf{w}})
$$

In global form

$$
\dot{\mathbf{U}}^{c v c}=\mathbf{R}(\mathbf{v}-\dot{\mathbf{w}})=\mathbf{R}(\mathbf{I}-\mathbf{X}) \mathbf{M}^{-1} \mathbf{p}
$$

where the coefficient matrix $\mathbf{R}$ depends on the element masses, the element internal energies, and the mesh coordinates. The flow work term depends on the pressure distribution $\hat{P}$, and has the general definition

$$
\dot{U}^{w r k(i)}=-\int_{V^{(i)}} \hat{P} \operatorname{div}(\hat{\mathbf{v}}) d V
$$

In discrete form

$$
\dot{U}^{w r k(i)}=V^{(i)} P^{(i)} \mathbf{g}^{(i) T} \mathbf{v}
$$

where $V^{(i)}$ is the volume of the $i^{\text {th }}$ element and $\mathbf{g}^{(i)}$ is a gradient operator which depends on the nodal coordinates. For the system

$$
\dot{\mathbf{U}}^{w r k}=\mathbf{G M}^{-1} \mathbf{p}
$$

where the coefficient matrix $\mathbf{G}$ is a function of the element (thermodynamic) pressures and the mesh coordinates.

The example simulations include a two term artificial viscosity. The viscous pressures $Q^{(i)}$ and $\sigma^{(i, j)}$ are computed as

$$
\begin{aligned}
Q^{(i)} & =c_{o} \rho_{o} c_{s} l_{e}<\mathbf{g}^{(i) T} \mathbf{v}> \\
\sigma^{(i, j)} & =\frac{c_{1} c_{s}}{\mathcal{A}^{(i, j)}}<\rho^{(i)}-\rho^{(i, j)}><\mathbf{a}^{(i, j) T} \mathbf{v}>
\end{aligned}
$$

where $c_{o}$ and $c_{1}$ are dimensionless constants, $c_{s}$ is a speed of sound, $l_{e}$ is a characteristic element length, $\rho_{o}$ is a reference density, $\mathcal{A}^{(i, j)}$ is the surface area common to elements ' $\mathrm{i}$ ' and ' $\mathrm{j}$ ', and the bracket function $\langle x\rangle$ is defined for any $x$ by

$$
<x>=x \hat{u}(x)
$$


with $\hat{u}$ the unit step function. The energy dissipation associated with this artificial viscosity is

$$
\dot{U}^{i r r(i)}=V^{(i)} Q^{(i)} \mathbf{g}^{(i) T}\left(\mathbf{M}^{-1} \mathbf{p}\right)+\sum_{j=1}^{n_{s}} \sigma^{(i, j)} \mathbf{a}^{(i, j) T}\left(\mathbf{M}^{-1} \mathbf{p}\right)
$$

hence for the system

$$
\dot{\mathbf{U}}^{i r r}=\mathbf{C M}^{-1} \mathbf{p}
$$

The viscous pressures are assumed to depend only on the total mass density, so that the coefficient matrix $\mathbf{C}$ is not a function of the species masses.

The example simulations also include a numerical heat conduction; for the $i^{\text {th }}$ element it is

$$
\dot{U}^{\operatorname{con}(i)}=\sum_{k=1}^{n_{s}} k^{(i, j)}\left(\theta^{(i)}-\theta^{(i, j)}\right)
$$

where $\theta^{(i, j)}$ is the temperature adjacent to surface $j$ of element $i$ and $k^{(i, j)}$ is a thermal conductivity

$$
k^{(i, j)}=k_{o} \rho_{o} c_{s} c_{v} \mathcal{A}^{(i, j)}
$$

with $k_{o}$ a dimensionless conduction coefficient and $c_{v}$ a reference specific heat. For the system

$$
\dot{\mathbf{U}}^{c o n}=\boldsymbol{\kappa}(\mathbf{m}, \mathbf{w}, \mathbf{s}, \mathbf{U}, t)
$$

where a time dependence may be introduced by the thermal boundary conditions applied to the system.

The complete internal energy evolution equations for the system are

$$
\dot{\mathbf{U}}=[\mathbf{R}(\mathbf{I}-\mathbf{X})+\mathbf{G}+\mathbf{C}] \mathbf{M}^{-1} \mathbf{p}-\boldsymbol{\kappa}
$$

\section{Virtual work}

In the Lagrange equation model developed here, a virtual work expression quantifies the external effects of loads on system boundaries and the internal effects of mesh motion. Since the nodal momenta are used as quasi-velocities $(\dot{\mathbf{q}}=\mathbf{p})$, the virtual work expression depends on virtual changes in the quasi-coordinates $\delta \mathbf{q}$ as well as virtual displacements of the finite element nodes $\delta \mathbf{w}$. The virtual work due to mechanical loads $(\mathbf{F})$ on closed external boundaries and total enthalpy $\left(h^{e x t(i, j)}\right)$ flux across open external boundaries is

$$
\delta W^{e x t}=\mathbf{F}^{T} \delta \mathbf{w}+\sum_{i=1}^{n_{e}} \sum_{j=1}^{n_{s}} h^{e x t(i, j)} \delta V^{(i, j)}
$$

where the enthalpy per unit volume includes total pressure $P^{e x t(i, j)}$ (thermodynamic and dynamic) and internal energy terms

$$
h^{e x t(i, j)}=P^{e x t(i, j)}+\rho^{(i, j)} e^{e x t(i, j)}
$$

and the virtual volume convected across element boundaries is

$$
\delta V^{(i, j)}=\mathbf{a}^{(i, j) T}\left(\mathbf{M}^{-1} \delta \mathbf{q}-\delta \mathbf{w}\right)
$$

Combining terms on the virtual displacement vectors,

$$
\delta W^{e x t}=\left[\mathbf{M}^{-1}\left(\mathbf{f}^{P}+\mathbf{f}^{e}\right)\right]^{T} \delta \mathbf{q}+\left(\mathbf{F}(t)-\mathbf{f}^{P}-\mathbf{f}^{e}\right)^{T} \delta \mathbf{w}
$$


where

$$
\mathbf{f}^{p}=\sum_{i=1}^{n_{e}} \sum_{j=1}^{n_{s}} P^{\operatorname{ext}(i, j)} \mathbf{a}^{(i, j)} \quad, \quad \mathbf{f}^{e}=\sum_{i=1}^{n_{e}} \sum_{j=1}^{n_{s}} \rho^{(i, j)} e^{\operatorname{ext}(i, j)} \mathbf{a}^{(i, j)}
$$

An additional 'internal' virtual work expression [2]

$$
\delta W^{i n t}=(\mathbf{L} \dot{\mathbf{w}})^{T} \delta \mathbf{q}-\left(\mathbf{L}^{T} \mathbf{p}\right)^{T} \delta \mathbf{w}
$$

balances the rate of change of kinetic energy, as quantified both in the elements and at the nodes, due to the mesh motion.

\section{Lagrange equations}

The canonical Lagrange equations for the system are

$$
\begin{gathered}
\frac{d}{d t}\left(\frac{\partial \mathcal{T}^{*}}{\partial \mathbf{p}}\right)=\mathbf{f} \\
-\frac{\partial \mathcal{T}^{*}}{\partial \mathbf{m}}=\mathbf{f}^{m} \\
\frac{\partial \mathcal{V}}{\partial \mathbf{U}}=\mathbf{f}^{U} \\
\frac{\partial \mathcal{V}}{\partial \mathbf{s}}=\mathbf{f}^{s} \\
\frac{\partial \mathcal{V}}{\partial \mathbf{w}}=\mathbf{f}^{w}
\end{gathered}
$$

where $\mathbf{f}, \mathbf{f}^{m}, \mathbf{f}^{U}, \mathbf{f}^{s}, \mathbf{f}^{w}$ are generalized forces due to the virtual work and the nonholonomic constraints. In this system the Lagrange equations for the generalized coordinates are degenerate (there are no generalized momenta associated with these coordinates) but nonetheless important, since they will determine in closed the Lagrange multipliers associated with the constraints. Introducing Lagrange multipliers $\boldsymbol{\lambda}^{m}, \boldsymbol{\lambda}^{U}, \boldsymbol{\lambda}^{s}, \boldsymbol{\lambda}^{w}$ for the nonholonomic constraints, and solving for the generalized forces,

$$
\begin{aligned}
\frac{d}{d t}\left(\frac{\partial \mathcal{T}^{*}}{\partial \mathbf{p}}\right)=\mathbf{L} \dot{\mathbf{w}}+\mathbf{M}^{-1}\left(\mathbf{f}^{P}+\mathbf{f}^{e}\right)-\mathbf{M}^{-1} \mathbf{X}^{T} \boldsymbol{\lambda}^{w}-\mathbf{M}^{-1}(\mathbf{G}+\mathbf{C})^{T} \boldsymbol{\lambda}^{U} & \\
- & \mathbf{M}^{-1}(\mathbf{I}-\mathbf{X})^{T}\left(\mathbf{A}^{T} \boldsymbol{\lambda}^{m}+\mathbf{Z}^{T} \boldsymbol{\lambda}^{s}+\mathbf{R}^{T} \boldsymbol{\lambda}^{U}\right)
\end{aligned}
$$

and

$$
\begin{aligned}
-\frac{\partial \mathcal{T}^{*}}{\partial \mathbf{m}} & =\mathbf{k}=\boldsymbol{\lambda}^{m} \\
\frac{\partial \mathcal{V}}{\partial \mathbf{U}} & =\mathbf{1}=\boldsymbol{\lambda}^{U} \\
\frac{\partial \mathcal{V}}{\partial \mathbf{s}} & =\mathbf{0}=\boldsymbol{\lambda}^{s} \\
\frac{\partial \mathcal{V}}{\partial \mathbf{w}} & =\mathbf{0}=\boldsymbol{\lambda}^{w}+\mathbf{F}(t)-\mathbf{f}^{P}-\mathbf{f}^{e}-\mathbf{L}^{T} \mathbf{M v}
\end{aligned}
$$


so that the final momentum balance equations are

$$
\begin{aligned}
& \dot{\mathbf{v}}=-\mathbf{M}^{-1}\left(\mathbf{X}^{T} \mathbf{L}^{T} \mathbf{M}-\right.\mathbf{M L X}) \mathbf{v} \\
&-\mathbf{M}^{-1}(\mathbf{I}-\mathbf{X})^{T}\left(\mathbf{A}^{T} \mathbf{k}-\mathbf{f}^{P}\right)+\mathbf{M}^{-1} \mathbf{X}^{T} \mathbf{F}(t) \\
&-\mathbf{M}^{-1}(\mathbf{G}+\mathbf{C})^{T} \mathbf{1}
\end{aligned}
$$

In the next section these momentum balance equations, supplemented by the nonholonomic constraints, are integrated to solve example problems in Lagrangian, Eulerian, and ALE frames.

\section{Example problems}

The example problems which follow include: (1) detonations in fluid and solid media, (2) stable and unstable detonations, (3) simple and relatively complex kinetics, and (4) comparisons with previous numerical work and with published experimental data.

\section{Example 1: thermal initiation of a detonation wave}

The first test problem, taken from Bihari and Schwendeman [33], models a binary mixture of reactant and product and a single irreversible reaction. The equation of state is

$$
P(\rho, e, z)=(\gamma-1) \rho(e+Q z) \quad, \quad \theta(e, z)=(e+Q z) / c_{v}
$$

where $z$ is a nondimensional progress variable (product mass fraction). The chemical heat release per unit mass $(Q)$ is 50 , the ratio of specific heats $(\gamma)$ is 1.4 , and $c_{v}=1 / \gamma$. The mass production rate is

$$
\omega(m, \theta, z)=m \kappa(1-z) \exp \left(\frac{-E}{\Re \theta}\right)
$$

with a rate constant $\kappa=42$, an activation energy $E=10$, and $\Re$ the universal gas constant. The initial conditions are everywhere $P=1, z=0, v=0$, with a mass density distribution

$$
\rho(x)=\frac{1}{1+3 \exp \left(-36(x+1)^{2}\right)}
$$

over the closed domain $x \in[-1,1]$. For the specified initial conditions, a high-temperature, lowdensity region is initially present on the left side of the modeled domain.

In this example the mesh is adaptive, with nodal velocities defined by

$$
\dot{w}^{(i)}=\frac{v^{(i-1)}+v^{(i+1)}}{2}
$$

The simulation was initialized with uniform mesh of 400 elements. Over the course of the simulation, a right-running shock wave develops; it is subsequently overtaken by a reaction wave, forming a detonation wave. Although there is no exact solution to this model problem, Figures 1-3 show that the pressure, density, and velocity distributions computed here agree closely with those of reference [33], the latter produced using a finite-volume method and an approximate Riemann solver on a much finer Eulerian mesh (2048 elements). Figure 4 shows that as the reaction wave develops the elements in the right side of the domain are compressed, to about one-third of their original size. Behind the wave, the elements are expanded to about three times their original size. Once the reaction wave overtakes the shock and the detonation spikes, the mesh is locally compressed by a factor as high as six. 
Example 2: mechanical initiation of a detonation wave

The second example (taken from reference [34]) models a moving boundary, and describes piston compression of a combustible mixture. The compression initiates a detonation wave in a binary system with an ideal gas reactant and product. The modeled reaction is

$$
\dot{z}=-K z<\theta-\theta_{c}>
$$

where $z$ is the reactant mass fraction, the rate coefficient $(K)$ is 1 , and the critical temperature $\left(\theta_{c}\right)$ is 1.3. The initial conditions are unit values for pressure and density, over the entire domain. The equation of state is

$$
P(\rho, e, z)=\rho(\gamma-1)(e-Q z) \quad, \quad \theta(e, z)=(e-Q z) / c_{v}
$$

with $\gamma=1.4, c_{v}=2.5$, and $Q=20$. The piston face is located initially at coordinate $w=1$, and moves with the constant velocity $v_{p}=-1$.

This problem was simulated using both a Lagrangian mesh and an ALE mesh, and demonstrates coupling of the mesh motion to a boundary velocity

$$
\dot{w}^{(i)}=\frac{w_{o}^{(i)}}{w_{o}^{(p)}} v_{p}
$$

where $w_{o}^{(i)}$ and $w_{o}^{(p)}$ denote the initial coordinates of the $i^{t h}$ node and the node attached to the piston face. The result is a mesh that compresses uniformly with the piston motion. Both the Lagrangian and ALE simulations employed 500 elements.

The simulation results for reacted mass fraction, temperature, pressure, density, and normalized cell size are shown in Figures 5 -10, at four different simulation times $(t=0.1,0.2,0.3,0.4)$. The ALE results are shown as solid lines, while the Lagrangian results are shown as dashed lines. The computed ALE and Lagrangian solutions are very similar, and both show good agreement with the solution of reference [34].

\section{Example 3: modeling stable and unstable detonations}

The third example problem is taken from reference [35], and compares stable and unstable detonations in an open system. Assuming a simple reaction mechanism, the minimum or ChapmanJouguet detonation velocity $\left(D_{C J}\right)$ of interest can be computed a-priori, by solving the RankineHugoniot equations $[14,16,22,35]$. Associated with any other detonation wavespeed $D>D_{C J}$ is an overdrive factor defined by

$$
f \equiv\left(\frac{D}{D_{C J}}\right)^{2}
$$

The stability of one-dimensional detonations has been shown to depend on the overdrive factor. In this example, we model first a detonation at $f=1.8$, known to be stable, and then model a detonation at $f=1.6$, known to be unstable $[15,22,35]$. The one-step, irreversible kinetic model is

$$
\omega(m, \theta, z)=m z K \exp \left(\frac{-50}{\theta}\right)
$$

where $z$ is a reactant mass fraction, $m$ is an element mass, and the rate constant $(K)$ is 145.69 for $\mathrm{f}=1.8$ or 230.75 for $\mathrm{f}=1.6$. The equation of state is

$$
P(\rho, e, z)=\rho \theta=(\gamma-1) \rho(e-Q z) \quad, \quad \theta(e, z)=(e-Q z) / c_{v}
$$


with $Q=50, \gamma=1.2$, and $c_{v}=1 /(\gamma-1)$, and the pre-shock conditions are $P=1$ and $\rho=1$. The computed Chapman-Jouguet detonation speed is 6.8095 [35]. In the stable detonation case $(\mathrm{f}=1.8)$, a spatial domain of width 100 is modeled with an Eulerian grid consisting of 8,000 uniform elements. In the unstable case $(\mathrm{f}=1.6)$, a spatial domain of width 200 is modeled with an Eulerian grid consisting of 16,000 uniform elements

In the stable detonation case $(\mathrm{f}=1.8)$, the simulation shows a shock speed of 9.136 , a shock pressure of 63.68 , a shock density of 4.016 , and a fluid velocity of 2.275 behind the shock. In the unstable detonation case $(\mathrm{f}=1.6)$ the the simulation shows a shock speed of $\mathrm{D}=8.613$, a shock pressure of 54.824 , a shock density of 3.643 , and a fluid velocity of 2.365 behind the shock. The time variation of the shock front pressure is shown in Figure 11, for the overdrive value of $f=1.8$. In this stable detonation, the pressure spikes to a value of 100 , then decays to a steady value of approximately 76 after time $t=50$. When the overdrive factor is reduced to $f=1.6$, the resulting detonation is unstable. The simulation shows a sustained galloping or pulsating shock front, without appreciable decay, as indicted by the shock front pressure-time history plotted in Figure 12. The simulation results produced here are in excellent agreement with those of Lian and $\mathrm{Xu}$ [35] and Hwang and co-workers [15], with regards to both amplitude and period of oscillation (mesh resolutions are identical). It should be noted that the modeled instability is not numerical, but rather physical, as indicated by both theory and experiment $[22,15]$.

\section{Example 4: detonation of a condensed phase explosive}

The fourth example considers the detonation of a condensed phase explosive, in two simulations modeling flyer plate impacts [36]. In the simulations, TNT blocks are impacted by thin mylar plates moving at $5.6 \mathrm{~km} / \mathrm{s}$. The TNT cylinder is $17.8 \mathrm{~mm}$ in length, and is modeled using 50 elements per millimeter. The mylar flyer plate is $0.254 \mathrm{~mm}$ in thickness and is modeled with 13 elements. In the first simulation: (1) the flyer plate impacts one end of the TNT block, (2) the detonation propagates through the explosive, and (3) the predicted surface velocity of a thin copper film deposited on the opposite end of the TNT block is compared with experiment. In the second simulation, (1) the TNT is impacted symmetrically by flyer plates striking each end, (2) the detonations propagate towards the center of the TNT block, and (3) the predicted time trace of the pressure wave reflected off the block centerline is compared to previous simulation work. These simulations use a Lagrangian grid.

Like some other widely used explosives, TNT (trinitrotoluene) is a relatively simple molecule $\left(\mathrm{C}_{7} \mathrm{H}_{5} \mathrm{~N}_{3} \mathrm{O}_{6}\right)$. Nonetheless a detailed detonation model would need to include many species and model many reactions. To reduce model complexity and computational cost, detonation simulations for real explosives are often accomplished by combing a two phase equation of state with a single stage 'ignition and growth' model of the reaction kinetics. Although both the equation of state and the reaction kinetics are empirical, their accuracy has been validated in well instrumented experiments [37, 38].

Following Clutter and Belk [36], the detonation simulations presented here employ a JonesWilkins-Lee $(J W L)$ equation of state for the TNT reactants and products $[37,36,38]$

$$
\begin{gathered}
P_{\text {solid }}(\rho, E)=17101\left(1-\frac{0.5675}{9.8 v}\right) e^{-9.8 v}-3.745\left(1-\frac{0.5675}{0.98 v}\right) e^{-0.98 v}+0.5675 \frac{E}{v} \\
P_{\text {gas }}(\rho, E)=673.1\left(1-\frac{0.3}{5.4 v}\right) e^{-5.4 v}+21.988\left(1-\frac{0.3}{1.8 v}\right) e^{-1.8 v}+0.3 \frac{E+7.0}{v}
\end{gathered}
$$

with $E=\rho e, v$ a relative volume ratio $\left(v=\rho_{o} / \rho\right)$, and $\rho_{o}=1.624 \mathrm{~g} / \mathrm{cc}$ the initial density of the solid explosive. For the inert mylar and copper, Gruneisen equations of state in the form

$$
P_{\text {mylar }}(\rho, E)=\frac{7.111 \mu(1+0.62 \mu)}{(1-0.56 \mu)^{2}}+0.76 E
$$




$$
P_{\text {copper }}(\rho, E)=\frac{138.625 \mu\left(1-0.01 \mu-0.235 \mu^{2}\right)}{(1-0.489 \mu)^{2}}+(2.02+0.47 \mu) E
$$

are assumed, where $\mu=\left(\rho / \rho_{o}\right)-1$ and the initial densities of the copper and mylar are 8.93 and $1.38 \mathrm{~g} / \mathrm{cc}$ respectively.

Over the course of the reaction, a mixture of solid reactant and gaseous product develops. The mixture pressure is computed using

$$
P=(1-F) P_{\text {solid }}+F P_{\text {gas }}
$$

where $\mathrm{F}$ is the mass fraction of the gaseous product. Since the value of $\mathrm{F}$ is initially zero, $\mathrm{F}$ also serves as a reaction progress variable, and is computed from an empirical kinetic ('ignition and growth') model [37, 36, 38]

$$
\frac{d F}{d t}=5.010^{7}(1-F)^{0.667}\left(\frac{\rho}{\rho_{o}}-1\right) \hat{u}(0.03-F)+3.610^{8}(1-F) F^{0.667} P^{1.2}
$$

The simulations conducted to model this example problem employed a flux-corrected transport (FCT) algorithm originally developed for application in finite difference methods [39], and adapted for use in finite element modeling of high-energy solids by Zhang and co-workers [40]. Use of a FCT algorithm on the Lagrangian mesh employed here is simple, because the nodal momentum correction depends only on nodal velocities. Note that the FCT algorithm is applied in integrating the discrete ODE model, the Lagrange equations developed in the preceding sections of the paper are not modified.

The simulation results for the single mylar flyer impact problem may be compared to experimental results reported by Kury and co-workers [38], as well as previous numerical modeling work reported by Clutter and Belk [36]. Here we simulate two cases: they compute the velocities of thin copper foils, 25 and 50 microns (12 and 24 elements) thick, attached to the end of the explosive block. The time history of the pressure evolution in the TNT block is shown in Figure 13. The initial impact pressure in the TNT peaks at $28 \mathrm{GPa}$, relaxes slightly, then rises gradually to a steady state value of $24 \mathrm{GPa}$; the latter results agree closely with the numerical simulations of Clutter and Belk [36]. The free-surface velocities for the copper foils are also shown in Figure 13: (1) the detonation wave reaches the foil at approximately 2.6 microseconds, and (2) the thinner foil is accelerated to a higher velocity than the thicker foil. The simulation results for the foil velocities show good agreement with the numerical simulation results of Clutter and Belk [36] and hence the experimental results of Kury and co-workers [38].

The pressure-time history in the TNT block subjected to symmetric impacts by two mylar flyer plates is shown in Figure 14; the simulation results are plotted at 0.1 microsecond intervals. The values for the peak internal pressure and the magnitude of the material dilatation at 4.8 microseconds after impact agree well with those of Clutter and Belk [36].

\section{Example 5: detonation of a hydrogen-air mixture}

The fifth example models the detonation of a hydrogen-air mixture. The simulation employs the equations of state and the reaction kinetics described by Powers and Paolucci [16]. The initial three-component mixture is $2 \mathrm{H}_{2}+\mathrm{O}_{2}+3.76 \mathrm{~N}_{2}$ and the modeled reactions produce six additional species: $\mathrm{H}_{2} \mathrm{O}, \mathrm{H}, \mathrm{O}, \mathrm{OH}, \mathrm{HO}_{2}$, and $\mathrm{H}_{2} \mathrm{O}_{2}$. The equation of state, representing a reacting mixture of thermally perfect gases with molecular masses $\mathfrak{M}_{k}$, is

$$
P\left(\rho, \theta, Y_{k=1 \ldots N_{s}}\right)=\rho \Re \theta \sum_{k=1}^{N_{s}} \frac{Y_{k}}{\mathfrak{M}_{k}}
$$




$$
e=\sum_{k=1}^{N_{s}} \frac{Y_{k}}{\mathfrak{M}_{k}}\left(h_{k}(\theta)-\Re \theta\right)
$$

where the temperature is determined implicitly, by balancing the sum of the species internal energies with the total mixture internal energy. The molar enthalpies of the species $h_{k}(\theta)$ and the species specific heats are assumed to be known functions of temperature [41].

The inflow (and initial pre-shock) conditions are assumed to be a stoichiometric mixture at one atmosphere and 298 degrees Kelvin. The kinetic model [16, 41] incorporates nine species and 57 simultaneous, reversible, temperature-dependent elementary reactions. The temperature dependence takes an Arrhenius functional form. The mass production rate for each species is obtained by standard methods [41].

The example problem simulates the development of a pulsating, overdriven hydrogen-air detonation. The detonation develops from an unreacted shock at 25 atmospheres and 1,400 degrees Kelvin, fed by a stoichiometric mixture flowing at 1,810 meters per second. The detonation products evolve to a variable outflow of mixed chemistry. The modeled spatial domain, $0.24 \mathrm{~cm}$ in length, was discretized using an Eulerian mesh of 1,200 uniform elements. Figures 15 and 16 show pressure and temperature profiles in the modeled region (initial conditions are represented on the right). Ten snapshots of the developing detonation are provided (the detonation moves right to left), and encompass two complete cycles of the pulsating detonation.

Simulated species mole fractions located downstream from the shock are plotted in Figure 17; they correspond to the pressure and temperature profiles plotted third from the left in Figures 15 and 16 (simulation time 5.298 microseconds). The evolved mole fractions for all species in the reaction zone, obtained from our dynamic simulation, are in excellent agreement with the estimated steady state distribution reported in reference [16].

\section{Conclusion}

The present paper extends Hamiltonian [2] and Lagrange equation [3] formulations of compressible fluid dynamics developed in previous work, introducing a method which incorporates both reacting media and ALE frames. Choosing internal energy and species masses as generalized coordinates, and separating the discretization and model formulation steps are the keys to extending discrete energy methods to reacting continuous media. Extension of the method to complex multidimensional flows is needed in order to address important engineering applications [42].

\section{Acknowledgements}

This research was supported by the National Science Foundation (CMS99-12475), the Texas Advanced Research Program (003658-0709-1999), NASA Johnson Space Center (NNX08AT06G), and the Office of Naval Research (N00014-05-1-0857).

\section{References}

[1] E.P. Fahrenthold, J.C. Koo, Discrete Hamilton's equations for viscous compressible fluid dynamics, Computer Methods in Applied Mechanics and Engineering 178 (1999) 1-22.

[2] J.C Koo, E.P. Fahrenthold, Discrete Hamilton's equations for arbitrary lagrangian-eulerian dynamics of viscous compressible flow, Computer Methods in Applied Mechanics and Engineering 189 (2000) 875-900. 
[3] E.P. Fahrenthold, C.R. Hean, Discrete Lagrange equations for thermofluid systems, Journal of Dynamic Systems, Measurement, and Control 130 (2008) 011009.

[4] S.P. Xiao, T. Belytschko, A bridging domain method for coupling continua with molecular dynamics, Computer Methods in Applied Mechanics and Engineering 193 (2004) 1645-1669.

[5] J.J. Beaman, A dynamic model of a pressure swing oxygen generation system, Journal of Dynamic Systems, Measurement, and Control 107 (1985) 111-116.

[6] J.K. Clutter, W. Shyy, Numerical methods for treating disparate scales in high-speed reacting flows, Numerical Heat Transfer, Part B 34 (1998) 165-189.

[7] R.P. Fedkiw, B. Merriman, S. Osher, High accuracy numerical methods for thermally perfect gas flows with chemistry, Journal of Computational Physics 132 (1997) 175-190.

[8] M. Valorani, D.A. Goussis, Explicit time-scale splitting algorithm for stiff problems: Autoignition of gaseous mixtures behind a steady shock, Journal of Computational Physics 169 (2001) 44-79.

[9] V.I. Lebedev, Explicit difference schemes with time-variable steps for solution of stiff systems of equations, in (L. Vuldov Ed.), Lecture Notes in Computer Science, Numerical Analysis and it applications. Springer-Verlag, Berlin, 1997.

[10] J.G. Verwer, Explicit Runge-Kutta methods for parabolic partial differential equations, Applied Numerical Mathematics, 22 (1996) 359.

[11] E. Loth, S. Sivier, J. Baum, Adaptive unstructured finite element method for two-dimensional detonation simulations, Shock Waves 8 (1998) 47-53.

[12] E.S. Oran, J.P. Boris, Numerical Simulation of Reactive Flow, Cambridge University Press, Cambridge, 2001.

[13] J.M. Powers, Review of multiscale modeling of detonation, Journal of Propulsion and Power 22 (2006) 1217-1229.

[14] K.K. Kuo, Principles of Combustion, John Wiley, New York, 1986.

[15] P. Hwang, R.P. Fedkiw, B. Merriman, T.D. Aslam, A.R. Karagozian, S.J. Osher, Numerical resolution of pulsating detonation waves, Combustion Theory and Modelling 4 (2000) 217-240.

[16] J.M. Powers, S. Paolucci, Accurate spatial resolution estimates for reactive supersonic flow with detailed chemistry, AIAA Journal 43 (2005) 1088-1099.

[17] A.K. Henrick, T.D. Aslam, J.M. Powers, Simulations of pulsating one-dimensional detonations with true fifth order accuracy. Journal of Computational Physics 213 (2006) 311-329.

[18] E.S. Oran, J.W. Weber, E.I. Stefaniw, M.H. Lefebvre, J.D. Anderson, A numerical study of a two-dimensional $\mathrm{H}_{2}-\mathrm{O}_{2}-\mathrm{Ar}$ detonation using a detailed chemical reaction model, Combustion and Flame 113 (1998) 147-163.

[19] E.F. Toro, Riemann Solvers and Numerical Methods for Fluid Dynamics, Springer-Verlag, Berlin, 1999.

[20] M. Ben-Artzi, The generalized Riemann problem for reactive flows, Journal of Computational Physics 81 (1996) 70-101. 
[21] A. Suresh, M.S. Liou, The Osher scheme for non-equilibrium reacting flows, International Journal for Numerical Methods in Fluids 15 (1992) 219-232.

[22] A. Bourlioux, A. Majda, V. Roytburd, Theoretical and numerical structure for unstable onedimensional detonations, SIAM Journal of Applied Mathematics 51 (1991) 303-343.

[23] V.T. Ton, Improved shock-capturing methods for multicomponent and reacting flows, Journal of Computational Physics 128 (1996) 237-253.

[24] M. Short, D. Stewart, Cellular detonation stability, part 1, Journal of Fluid Mechanics 368 (1998) 229-262.

[25] A. Bourlioux, A. Majda, Theoretical and numerical structure of unstable detonations, Philosophical Transactions of the Royal Society of London A 350 (1995) 29-68.

[26] S. Yungster, K. Radhakrishnan, Pulsating one-dimensional detonations in hydrogen-air mixtures, Combustion Theory and Modelling 8 (2004) 745-770.

[27] Y. Daimon, A. Matsuo, Unsteady features on one-dimensional hydrogen-air detonations, Physics of Fluids 19 (2007) 116101.

[28] S. Singh, Y. Rastigejev, S. Paolucci, J.M. Powers, Viscous detonation in $\mathrm{H}_{2}-\mathrm{O}_{2}-\mathrm{Ar}$ using intrinsic low-dimensional manifolds and wavelet adaptive multilevel representation, Combustion Theory and Modelling 5 (2001) 163-184.

[29] J.H. Ginsberg, Advanced Engineering Dynamics, Cambridge University Press, Cambridge, 1998.

[30] H. Baruh, Analytical Dynamics, WCG/McGraw-Hill, Boston, 1999.

[31] F.T. Brown, Hamiltonian and lagrangian bond graphs, Journal of the Franklin Institute 328 (1991) 809-831.

[32] H.B. Callen, Thermodynamics and an Introduction to Thermostatistics, John Wiley, New York, 1985.

[33] B.L. Bihari, D. Schwendeman, Multiresolution schemes for the reactive Euler equations, Journal of Computational Physics 154 (1999) 197-230.

[34] T. Lappas, A. Leonard, P. Dimotakis, An adapative lagrangian method for computing 1-D reacting and non-reacting flows, Journal of Computational Physics 104 (1993) 361-376.

[35] Y.S. Lian, K. Xu, A gas-kinetic scheme for multimaterial flows and its application in chemical reactions, Journal of Computational Physics 163 (2000) 349-375.

[36] J.K. Clutter, D. Belk, Simulation of detonation wave interaction using an ignition and growth model, Shock Waves 12 (2002) 251-263.

[37] E.L. Lee, C.M. Tarver, Phenomenological model of shock initiation in heterogeneous explosives, Physics of Fluids 23 (1980) 2362-2372.

[38] J.W. Kury, R.D. Breithaupt, C.M. Tarver, Detonation waves in trinitrotoluene, Shock Waves 9 (1999) 227-237.

[39] J.P. Boris, D.L. Book, Flux-corrected transport I: SHASTA, a fluid transport algorithm that works, Journal of Computational Physics 11 (1973) 38-69. 
[40] J. Zhang, Z. Duan, J. Ding, Simulating shock to detonation transition: algorithm and results, Journal of Computational Physics 150 (1999) 128-142.

[41] C.R. Hean, Discrete Lagrange equations for reacting thermofluid systems. Ph.D. dissertation, University of Texas at Austin, 2009.

[42] T. Plewa, T.J. Linde, V.G. Weirs, Adaptive mesh refinement, theory and applications, in Proceedings of the Chicago Workshop on Adaptive Mesh Refinement Methods, Springer-Verlag, Berlin, 2005. 


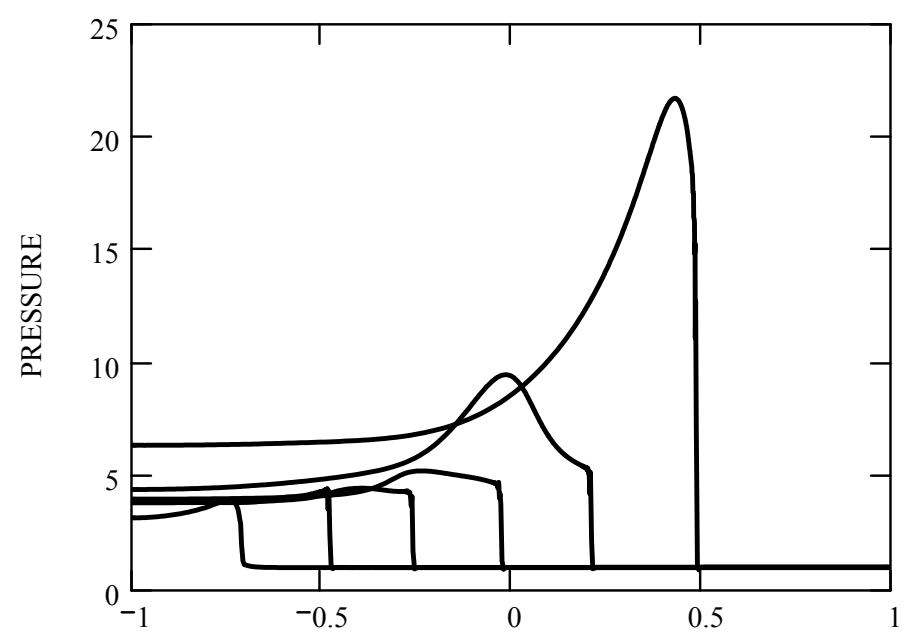

Figure 1: Transient pressure history of developing detonation $(\mathrm{t}=0.1,0.2,0.3,0.4,0.5,0.6, \mathrm{l}-\mathrm{r})$.

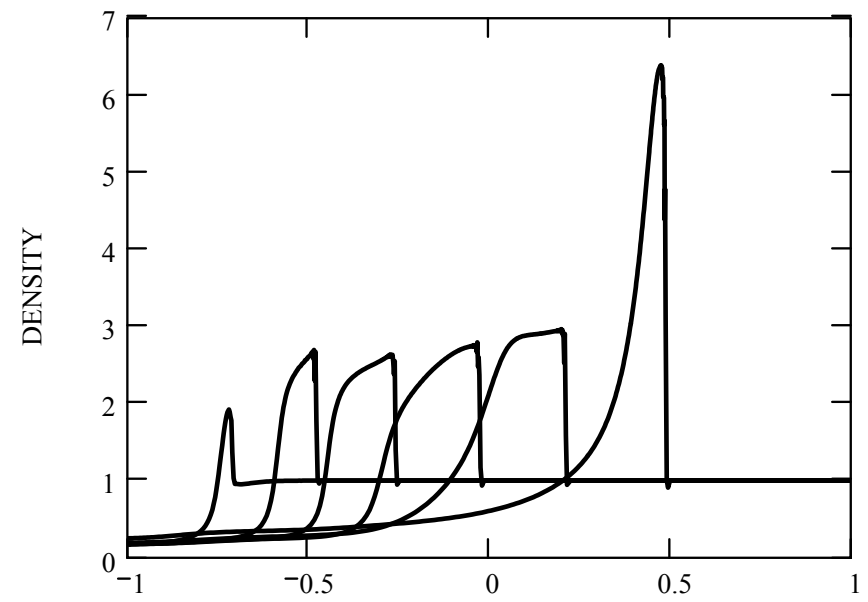

Figure 2: Transient density history of developing detonation $(\mathrm{t}=0.1,0.2,0.3,0.4,0.5,0.6,1-\mathrm{r})$. 


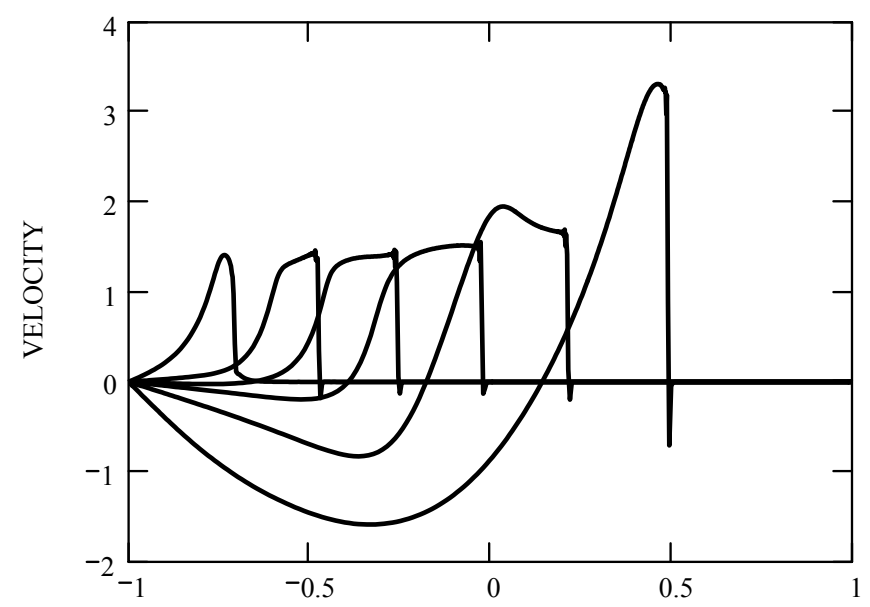

Figure 3: Fluid velocity history of developing detonation $(\mathrm{t}=0.1,0.2,0.3,0.4,0.5,0.6, \mathrm{l}-\mathrm{r})$.

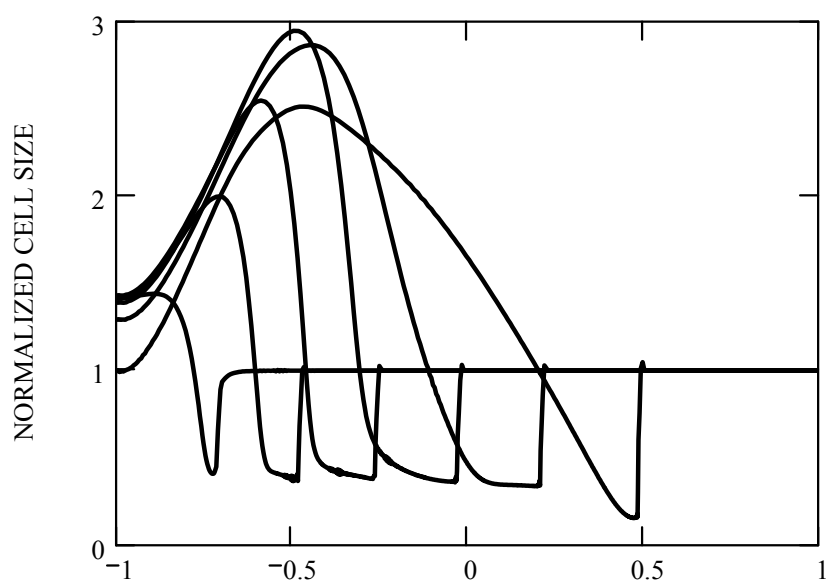

Figure 4: Cell size history of developing detonation $(\mathrm{t}=0.1,0.2,0.3,0.4,0.5,0.6, \mathrm{l}-\mathrm{r})$. 


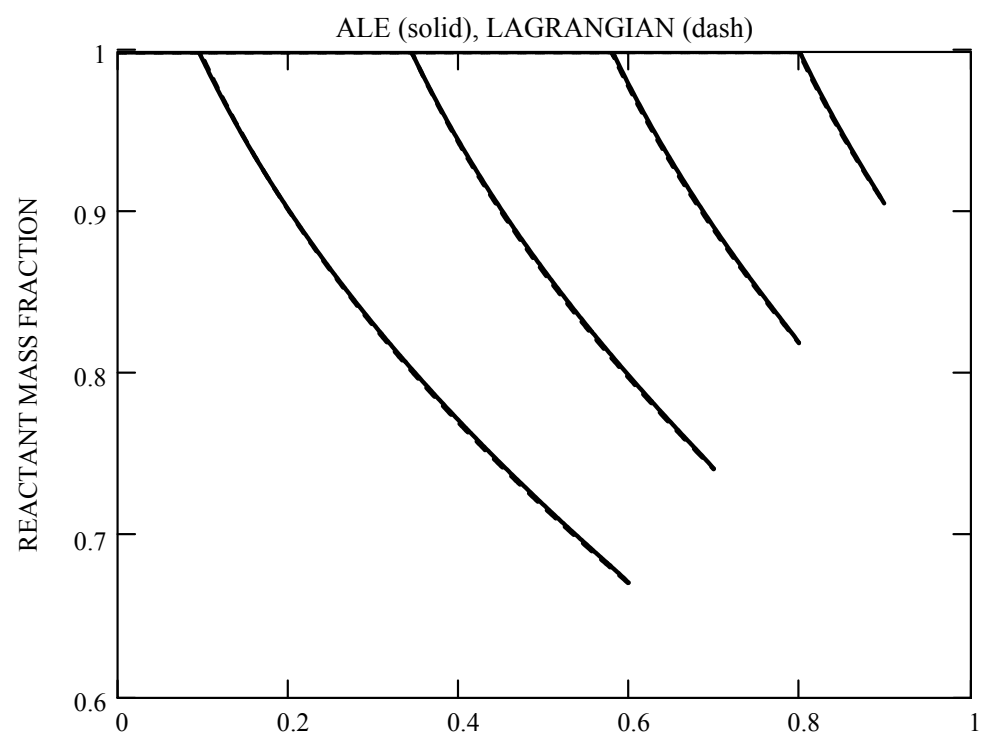

Figure 5: Mass-fraction during piston detonation $(\mathrm{t}=0.1,0.2,0.3,0.4, \mathrm{r}-\mathrm{l})$.

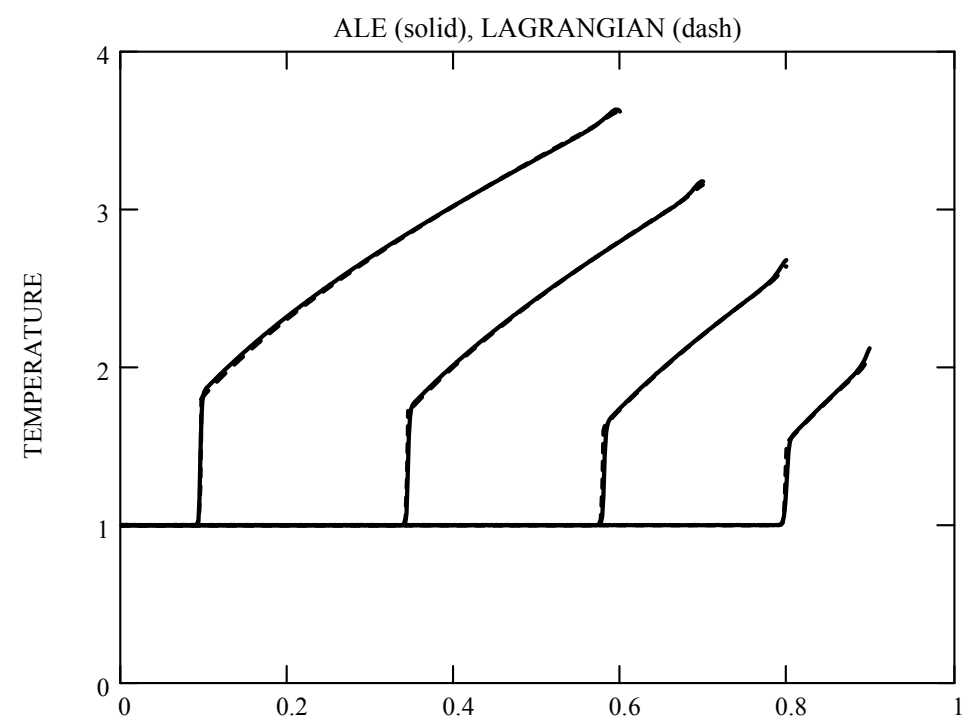

Figure 6: Temperature during piston detonation $(\mathrm{t}=0.1,0.2,0.3,0.4, \mathrm{r}-\mathrm{l})$. 


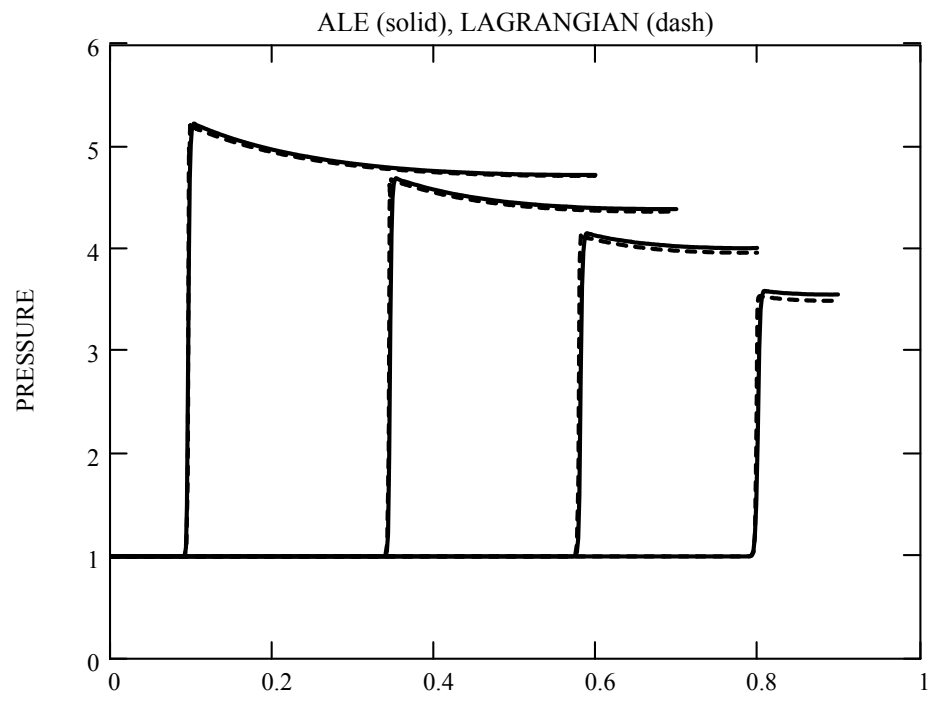

Figure 7: Pressure during piston detonation $(\mathrm{t}=0.1,0.2,0.3,0.4, \mathrm{r}-\mathrm{l})$.

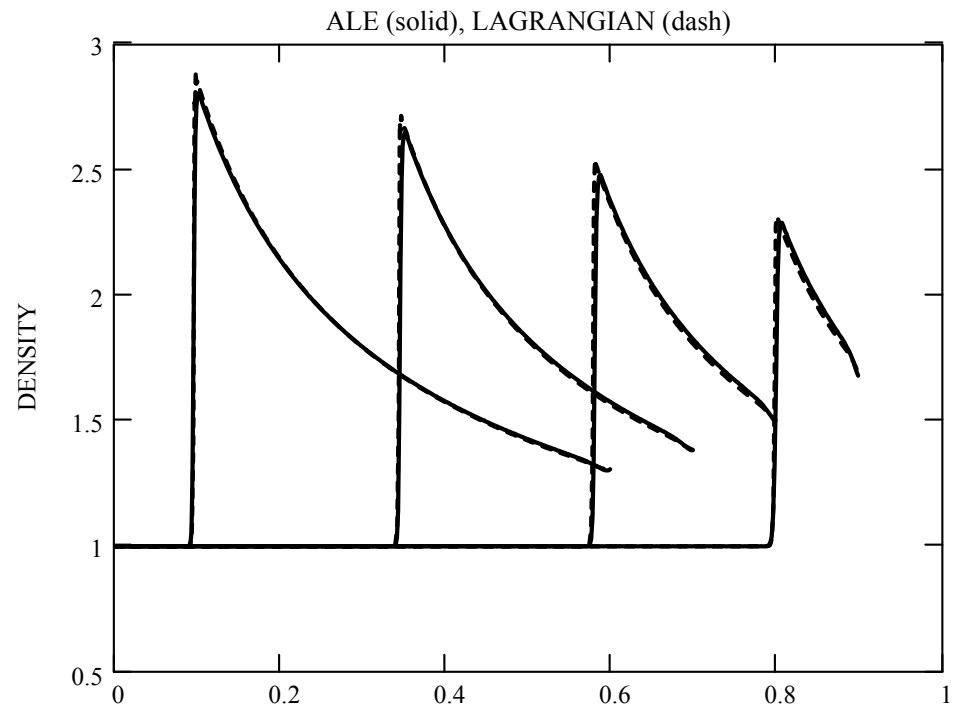

Figure 8: Density during piston detonation $(\mathrm{t}=0.1,0.2,0.3,0.4, \mathrm{r}-\mathrm{l})$. 


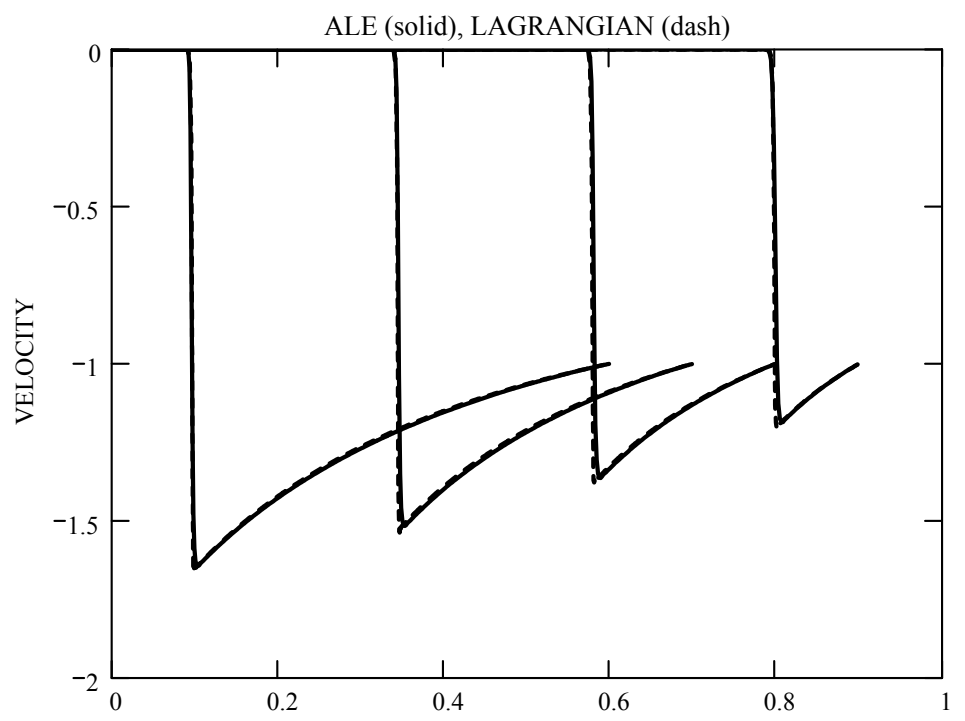

Figure 9: Fluid velocity during piston detonation $(\mathrm{t}=0.1,0.2,0.3,0.4, \mathrm{r}-\mathrm{l})$.

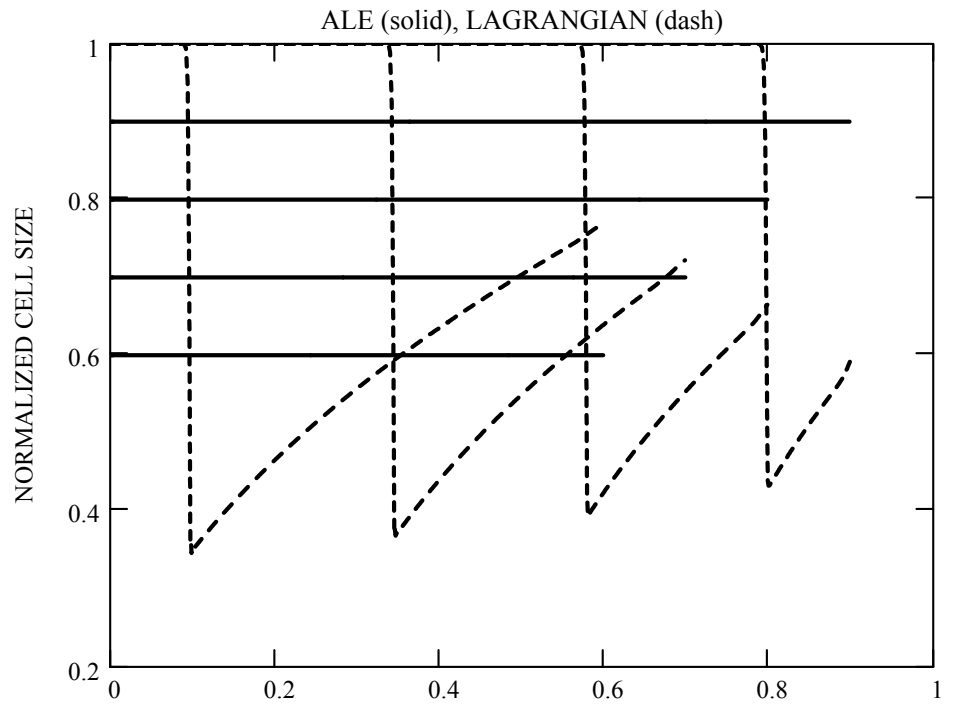

Figure 10: Cell size during piston detonation $(\mathrm{t}=0.1,0.2,0.3,0.4, \mathrm{r}-\mathrm{l})$. 


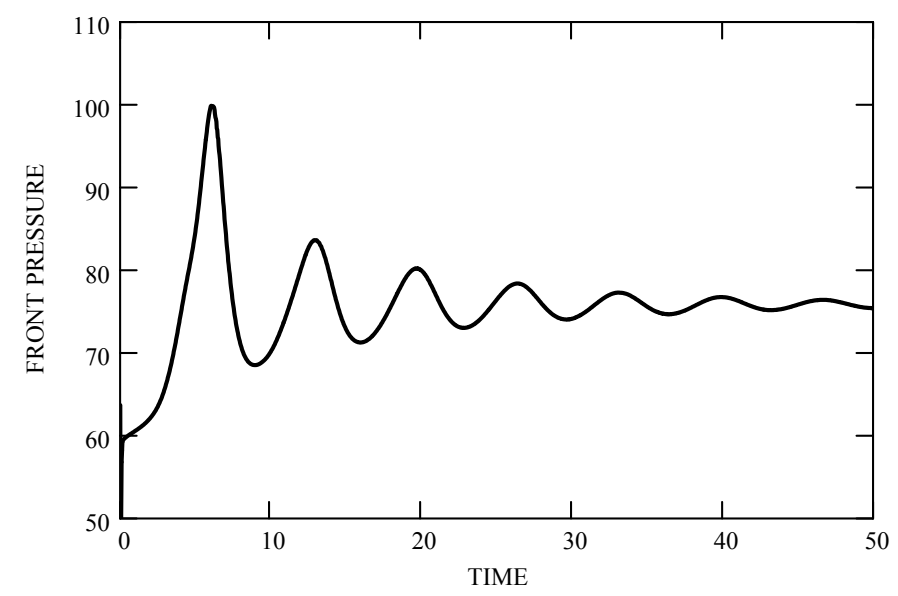

Figure 11: Pressure history at shock front of stable overdriven detonation wave, $f=1.8$.

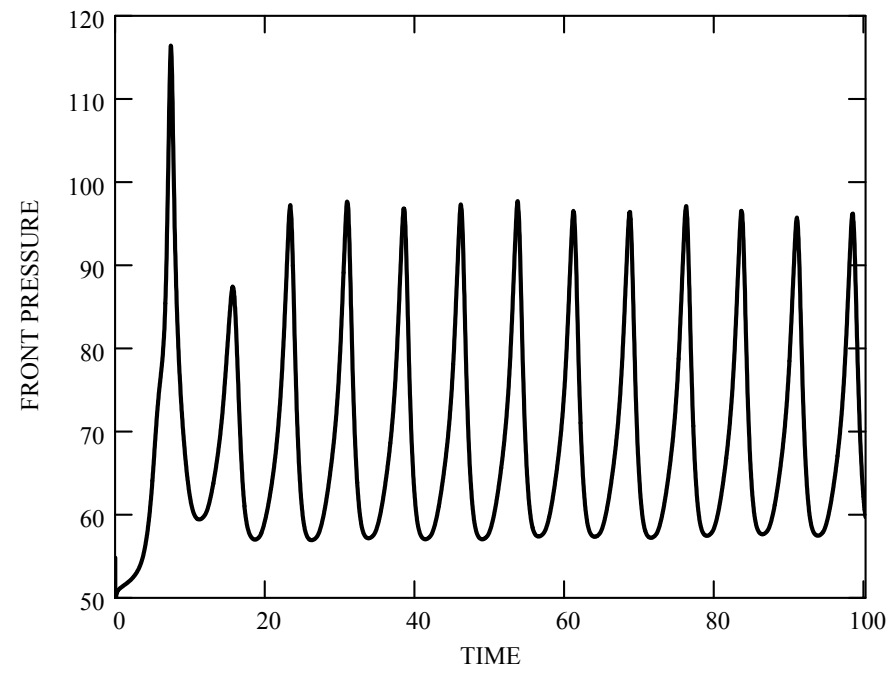

Figure 12: Pressure history at shock front of pulsating overdriven detonation wave, $f=1.6$. 

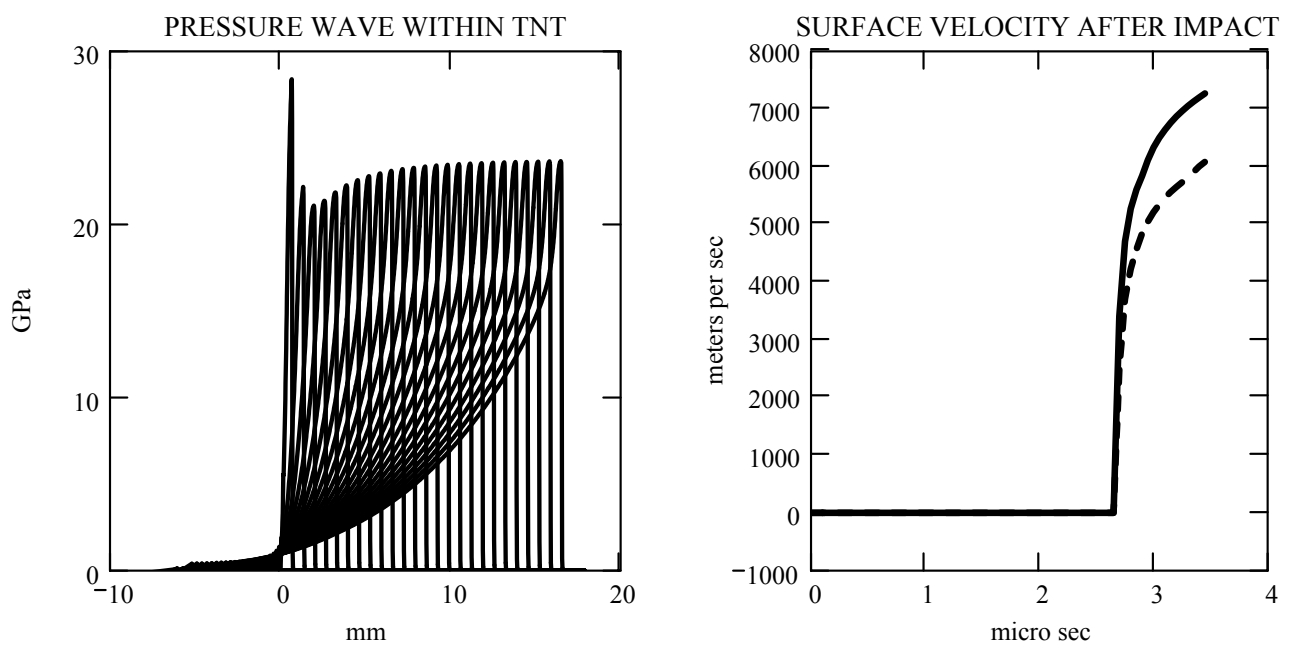

-25 micron $\mathrm{Cu}$
$-\quad 50$ micron $\mathrm{Cu}$

Figure 13: TNT pressure history and free surface velocity following single impact of mylar flyer .

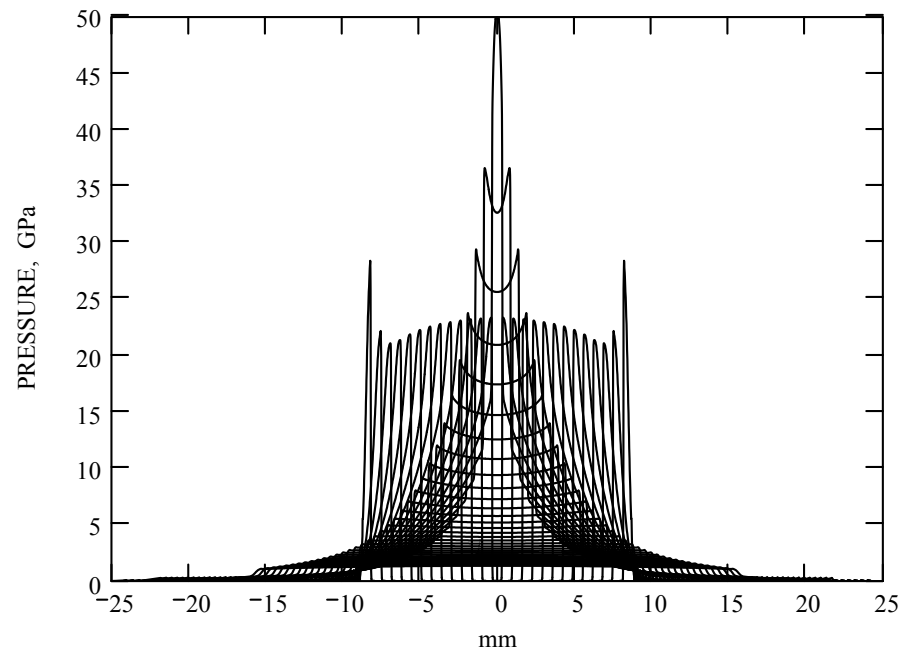

Figure 14: Pressure history of TNT detonation following symmetric impact of mylar flyers (elapsed time $4.8 \mu$ sec). 


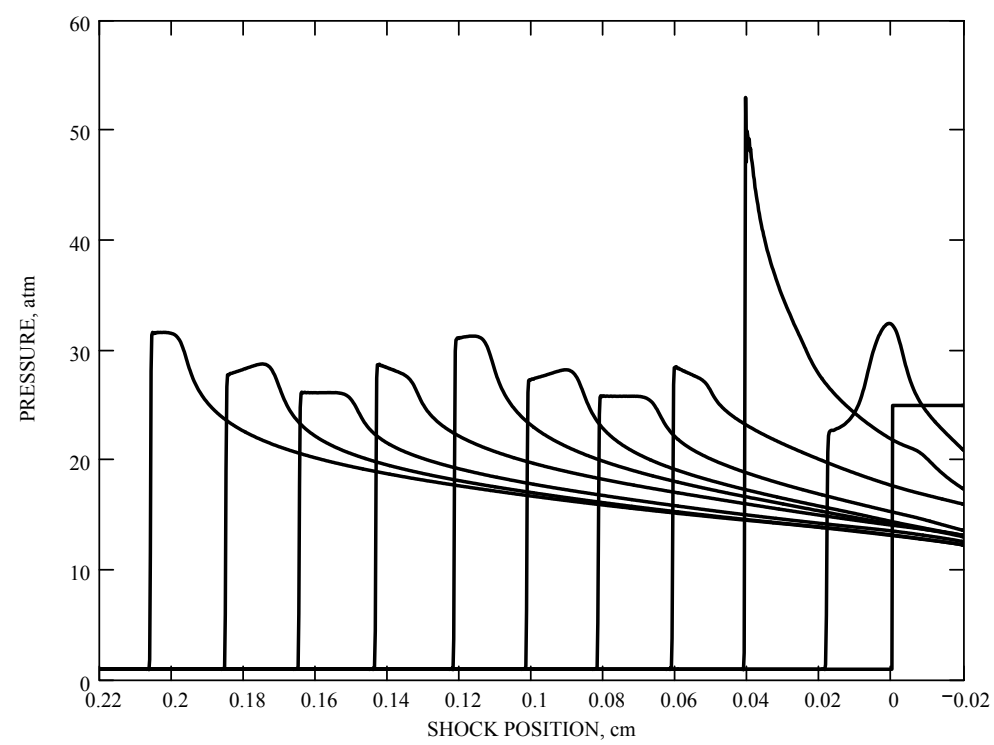

Figure 15: Pressure profiles of pulsating hydrogen-air detonation, moving right to left, total elapsed time $6.59 \mu$ sec.

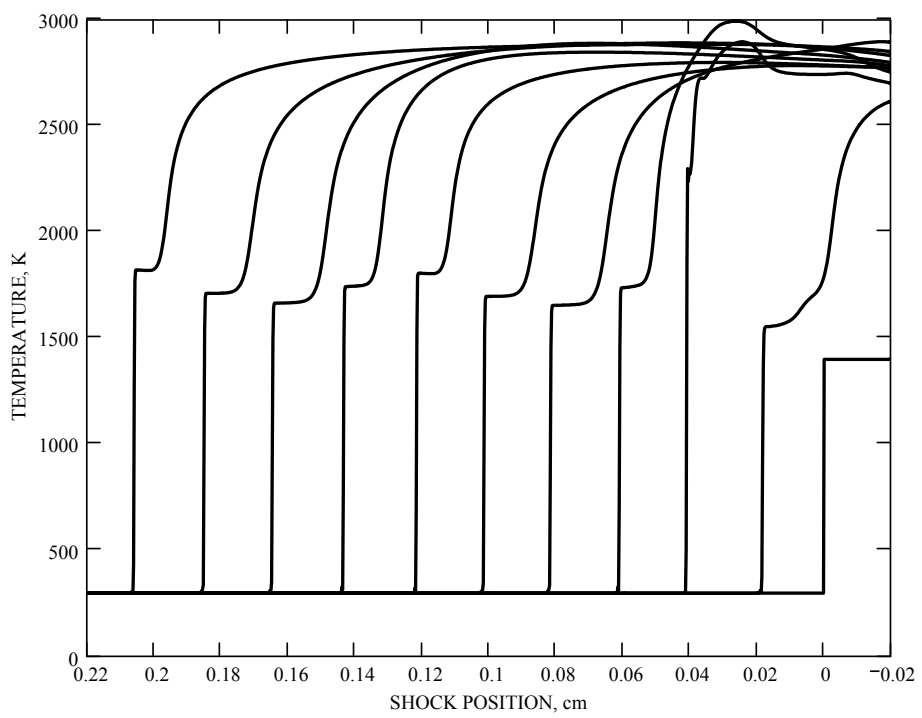

Figure 16: Temperature profiles of pulsating hydrogen-air detonation, moving right to left, total elapsed time $6.59 \mu$ sec. 


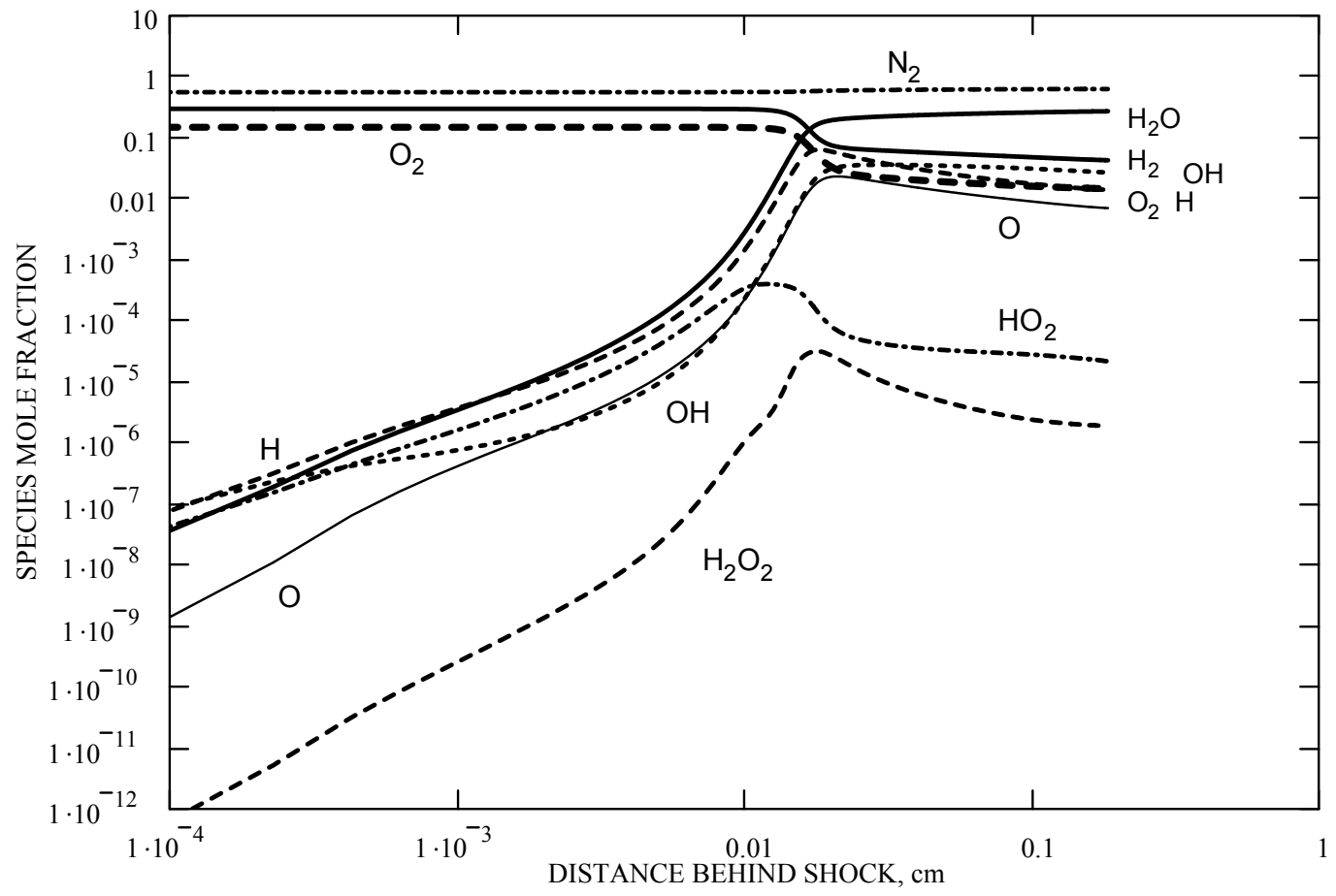

Figure 17: Species mole fractions downstream of shock front, at $\mathrm{t}=5.298 \mu$ sec. 